\begin{tabular}{|c|c|}
\hline Title & Fluidic Sensors Based on Photonic Crystal Fiber Gratings: Impact of the A mbient Temperature \\
\hline Author(s) & Florous, Nikolaos John; Saitoh, Kunimasa; V arsheney, Shailendra K.; Koshiba, Masanori \\
\hline Citation & $\begin{array}{l}\text { IEEE Photonics T echnology Letters, 18(21), 2206-2208 } \\
\text { https://doi.org/10.1109/LPT.2006.884275 }\end{array}$ \\
\hline Issue Date & 2006 \\
\hline Doc URL & http:/hdl.handle.net/2115/17191 \\
\hline Rights & $\begin{array}{l}\text { "@ } 2006 \text { IEEE. Personal use of this material is permitted. However, permission to reprint/republish this material for } \\
\text { advertising or promotional purposes or for creating new collective works for resale or redistribution to servers or lists, } \\
\text { or to reuse any copyrighted component of this work in other works must be obtained from the IEEE." IEEE, IEEE } \\
\text { Photonics Technology Letters, vol. 18, issue 21, 2006, pp. 2206-2208 }\end{array}$ \\
\hline Tyре & article \\
\hline File Information & IEEE-PTL-18-21.pdf \\
\hline
\end{tabular}

Instructions for use 


\title{
Fluidic Sensors Based on Photonic Crystal Fiber Gratings: Impact of the Ambient Temperature
}

\author{
Nikolaos John Florous, Member, IEEE, Kunimasa Saitoh, Member, IEEE, Shailendra K. Varsheney, Member, IEEE, \\ and Masanori Koshiba, Fellow, IEEE
}

\begin{abstract}
The spectral and thermooptical responses of photonic crystal fiber gratings (PCFGs) infiltrated with gaseous solutions, are investigated theoretically using an accurate semivectorial modal solver combined with exact equations for the reflection response of fiber Bragg gratings. We demonstrate numerically perhaps for the first time, that by an appropriate selection of the design parameters, it is possible to obtain compact sensing platforms based on the shift of the calculated reflectance spectra of the PCFG. Thus, our investigation adds evidence to the potential use of PCFGs as gas/liquid sensors. In addition, we show through a thermooptical sensitivity analysis that this type of sensors can easily meet the requirements for channel allocation in wavelength-division-multiplexing systems with relatively low temperature sensitivity.
\end{abstract}

Index Terms-Bragg gratings, gas sensors, photonic crystal fiber (PCF), sensitivity analysis, temperature-dependent Sellmeier equations, virtual boundary method.

\section{INTRODUCTION}

A $S$ telecommunication traffic increases due to the rapid growth in the use of telephone, television, data transmission, and the Internet, the need for communication systems that can handle more and more information increases rapidly all over the world. Accordingly, more channels over wider bandwidths are required to fulfill the increased demand. Wavelength-division multiplexing (WDM) [1] to process multiple communication channels together in an optical link has been established as a promising solution for the increased capacity. The WDM technique allows long-haul point-to-point lightwave transmission systems to provide multiple channels simultaneously in the $1.55-\mu \mathrm{m}$ wavelength regime.

In a WDM communication system, one of the most essential components is the optical filter. Incorporating Bragg gratings in optical fibers, application-specific optical filters can be designed, based on the well-developed grating theory [2]. The main drawback of incorporating conventional fiber Bragg gratings (FBGs), for realizing such optical components, not only for telecommunication applications but also as sensing platforms, is their high thermal sensitivity [3] as well as the very few degrees of freedom in engineering their modal properties. These two factors can significantly limit the potential capabilities of FBGs, especially in the sensor industry. Thus, other ultimate technologies have to be introduced to overpass these difficulties.

Manuscript received June 1, 2006; revised August 13, 2006.

The authors are with the Division of Media and Network Technologies, Hokkaido University, Sapporo 060-0814, Japan (e-mail: nflorous@dpo7.ice. eng.hokudai.ac.jp)

Color versions of Figs. 1-4 are available online at http://ieeexplore.ieee.org. Digital Object Identifier 10.1109/LPT.2006.884275

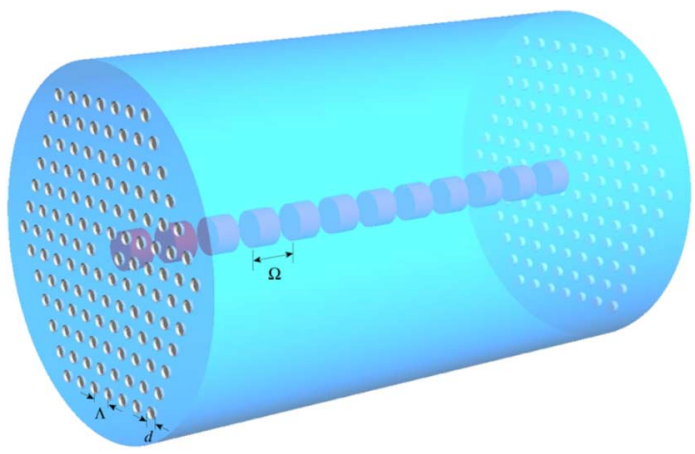

Fig. 1. Schematic cross section of the proposed PCFG considered as a fluidic sensor. The microstructured holes are arranged in a hexagonal configuration with lattice constant $\Lambda$ and diameters- $d$. The period of the grating perturbation is $\Omega$.

It has been conjectured that photonic crystal fibers (PCFs), also known as holey fibers (HFs) or microstructure optical fibers, are promising platforms for many novel applications in the telecommunication industry, as well as in the traditional sensor industry, because they can enable light to be controlled in the fiber in ways not previously possible or even imaginable. They have attracted considerable attention in recent years [4] due to the unlimited possibilities in engineering their modal properties. Despite their exceptional characteristics, there are no complete scientific reports incorporating PCF gratings (PCFGs) as sensors [5], mainly for two reasons. At first, a robust numerical analysis which permits the theoretical prediction of the thermooptical response in PCFGs is not known. On the other hand, temperature-independent Sellmeier equations were used so far to describe their material dispersion properties, not allowing the temperature-dependent phenomena to be studied, especially when PCFGs with infiltrated gaseous and/or liquid solutions are considered.

Taking all of the above circumstances into account, we devote the present letter to describe the first complete theoretical investigation of the spectral as well as the thermooptical responses in PCFG gas sensors, and we identify the true impact of the ambient temperature fluctuation to the spectral response of PCFGs sensors, which is found to be much lower than that of conventional FBGs [3], thus enabling the preeminent use of PCFGs for various sensor applications.

\section{Modeling the SPeCtRAL Response In PCFGs}

Consider the PCFG device as shown in Fig. 1. The background material is considered to be pure silica $\left(\mathrm{SiO}_{2}\right)$, while the microstructured holes, arranged in a hexagonal configuration with lattice constant $\Lambda$ and hole-diameters $d$, are infiltrated with gaseous solutions. In particular, we will consider the 
following gases: dry air with standard volumetric composition of $78.03 \%$ nitrogen $(\mathrm{N}), 20.99 \%$ oxygen $\left(\mathrm{O}_{2}\right), 0.03 \%$ carbon dioxide $\left(\mathrm{CO}_{2}\right), 0.01 \%$ hydrogen $\left(\mathrm{H}_{2}\right)$, and $0.94 \%$ argon $(\mathrm{Ar})$, a noble gas solution consisting of argon (Ar), carbon dioxide $\left(\mathrm{CO}_{2}\right)$, and n-butane $\left(\mathrm{C}_{4} \mathrm{H}_{10}\right)$. The PCFG is considered longitudinally invariant, while a perturbation of the refractive index is introduced in the core region with the following expression:

$$
\varepsilon=\varepsilon_{s}+\Delta \varepsilon(z) \cong n_{s}^{2}+2 n_{s} \delta n_{\mathrm{eff}}(z)
$$

where $n_{s}$ stands for the refractive index of pure silica, with a temperature-dependence of the following type [6], [7]:

$$
n_{s}^{2}=A+\frac{B \lambda^{2}}{\lambda^{2}-C}+\frac{D \lambda^{2}}{\lambda^{2}-E}
$$

where the constants $A, B, C, D$, and $E$ are linearly dependent on temperature $T$ (in Celsius) in the following manner:

$$
\begin{aligned}
& A=M_{A} T+Y_{A} ; M_{A}=3.1463 \times 10^{-8} ; Y_{A}=1.3855 \\
& B=M_{B} T+Y_{B} ; M_{B}=2.0427 \times 10^{-5} ; Y_{B}=0.7884 \\
& C=M_{C} T+Y_{C} ; M_{C}=2.8155 \times 10^{-6} ; Y_{C}=0.011029 \\
& D=M_{D} T+Y_{D} ; M_{D}=-6.7886 \times 10^{-5} ; Y_{D}=0.91136 \\
& E=M_{E} T+Y_{E} ; M_{E}=100 ; Y_{E}=10^{-10} .
\end{aligned}
$$

The modulation term $\delta n_{\mathrm{eff}}(z)$ is given as

$$
\delta n_{\mathrm{eff}}(z)=\overline{\delta n_{\mathrm{eff}}}\left\{1+v \cos \left(\frac{2 \pi}{\Omega} z\right)\right\}
$$

with $\overline{\delta n_{\text {eff }}}$ being the averaged refractive index change over one period, $v$ is the fringe visibility of the index change, $\Omega$ is the period of the grating perturbation, and $z$ is the propagation distance. If the grating is assumed uniform along the light propagation direction, $\overline{\delta n_{\mathrm{eff}}}$ is a constant. For a single-mode Bragg reflection grating, a "dc" (period-averaged) coupling coefficient $\sigma$ and an "ac" coupling coefficient $\kappa$ [2] can be found by the following simple relations:

$$
\sigma=\frac{2 \pi}{\lambda} \overline{\delta n_{\mathrm{eff}}}, \quad \kappa=\frac{\pi}{\lambda} v \overline{\delta n_{\mathrm{eff}}} .
$$

In our case, these coupling coefficients are simple constants. The closed-form solution for the power reflection coefficient $R(\lambda)$ of a uniform fiber grating of length $z=L$ is found to be

$$
R(\lambda)=\frac{\sinh ^{2}\left(L \sqrt{\kappa^{2}-\hat{\sigma}^{2}}\right)}{\cosh ^{2}\left(L \sqrt{\kappa^{2}-\hat{\sigma}^{2}}\right)-\frac{\hat{\sigma}^{2}}{\kappa^{2}}}
$$

where $\lambda$ is the operational wavelength and $\hat{\sigma}$ is a "dc" self-coupling coefficient defined as

$$
\hat{\sigma}=\sigma+\delta=\frac{2 \pi}{\lambda} \overline{\delta n_{\mathrm{eff}}}+2 \pi n_{\mathrm{eff}}\left(\frac{1}{\lambda}-\frac{1}{2 n_{\mathrm{eff}} \Omega}\right) .
$$

The Sellmeier equations for different gaseous solutions considered here follow a Lorenz-Lorenz type of wavelength and temperature dependence, which for the particular cases of dry air and carbon dioxide take the following form [8]:

$$
\begin{aligned}
n_{\text {Air }}(T)= & 1+10^{-8}\left(6432.8+\frac{2949810 \lambda^{2}}{146 \lambda^{2}-1}+\frac{25540 \lambda^{2}}{41 \lambda^{2}-1}\right) \\
& \times\left(\frac{1+a T_{s}}{1+a T}\right) \frac{P}{P_{s}}
\end{aligned}
$$

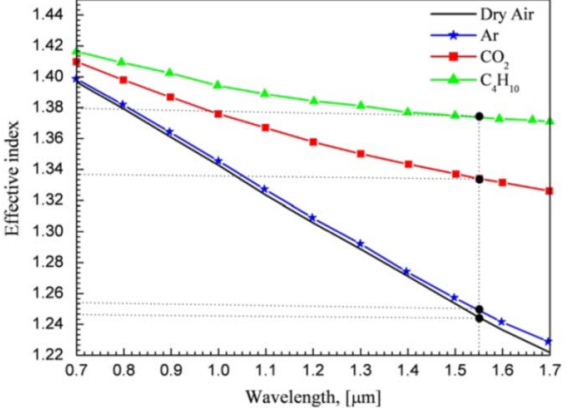

Fig. 2. Effective refractive indexes as a function of the operational wavelength $\lambda$ in micrometers, for dry air (solid black line), argon (blue line, stars), carbon dioxide (red line, squares), and n-butane (green line, triangles), at $T=27^{\circ} \mathrm{C}$. Notice that the effective index shifts to higher values for gaseous solutions with higher mass densities.

$$
\begin{aligned}
& n_{\mathrm{CO}_{2}}(T)= 1.38+10^{-8}\left(22822.1+\frac{117.8}{\lambda^{2}}\right. \\
&\left.+\frac{2406030 \lambda^{2}}{130 \lambda^{2}-1}+\frac{15997 \lambda^{2}}{38.9 \lambda^{2}-1}\right) \\
& \times\left(\frac{1+a^{\prime} T_{s}}{1+a^{\prime} T}\right) \frac{P}{P_{s}}
\end{aligned}
$$

where in the above expressions, $T$ is the temperature in Celsius, $T_{s}=15^{\circ} \mathrm{C}$ is a reference temperature, $\lambda$ is the wavelength in $\mu \mathrm{m}, P$ is the pressure in millimeter $(\mathrm{mm})-\mathrm{Hg}, P_{s}=760 \mathrm{~mm}-\mathrm{Hg}$ is the reference standard pressure under normal conditions, $a$ and $a^{\prime}$ are slowly varying with temperature parameters, which can be depicted from [8], for different wavelength bands. Similar equations can be written for the other two gases considered here, that is argon (Ar) and n-butane $\left(\mathrm{C}_{4} \mathrm{H}_{10}\right)$ [9]. The typical temperature interval, within which relations (2), (7a), and (7b) give accurate results, ranges from $-20^{\circ} \mathrm{C}$ up to $100^{\circ} \mathrm{C}$.

\section{SeleCted Numerical Results AND Discussion}

Fig. 2 shows the calculated effective refractive indexes of the fundamental mode, for dry air (solid black line), argon (blue line, stars), carbon dioxide (red line, squares), and n-butane (green line, triangles), as a function of the wavelength $\lambda$. The pitch constant $\Lambda$ is chosen as $1.0 \mu \mathrm{m}$ and the diameter of the holes is $0.8 \mu \mathrm{m}$, in order to accommodate more optical functionality around $1.55-\mu \mathrm{m}$ wavelength regime. Moreover, an induced refractive index change of $\overline{\delta n_{\text {eff }}}=8 \times 10^{-4}$, a visibility $\nu=0.02$, and a uniform grating length $z=L=70 \mathrm{~mm}$ are considered. Few researches have recently reported on HFs that are filled with liquid or solid-like polymers [10]. However, extensive survey reveals that there is no attempt on grating-incorporated HFs filled with different gases. For the purpose of calculation of the reflectivity spectrum $R(\lambda)$ of the proposed PCFG, the effective refractive indexes for four different cases are obtained by the virtual boundary method approach [11], taking into account the material dispersion effects. Once the effective refractive indexes (1.2441 for dry air, 1.2482 for Ar, 1.3287 for $\mathrm{CO}_{2}$, and 1.3803 for $\left.\mathrm{C}_{4} \mathrm{H}_{10}\right)$ are obtained at the desired wavelength of $\lambda=1.55 \mu \mathrm{m}$, shown as black dots in Fig. 2, the reflectivity spectrum can be calculated by using (5). The reflectivity spectra are plotted in Fig. 3 for the cases when the five-layer holes are filled with dry air (black solid line), argon-Ar (blue line stars), carbon dioxide- $\mathrm{CO}_{2}$ (red line squares), or n-butane (green line triangles). From the results 


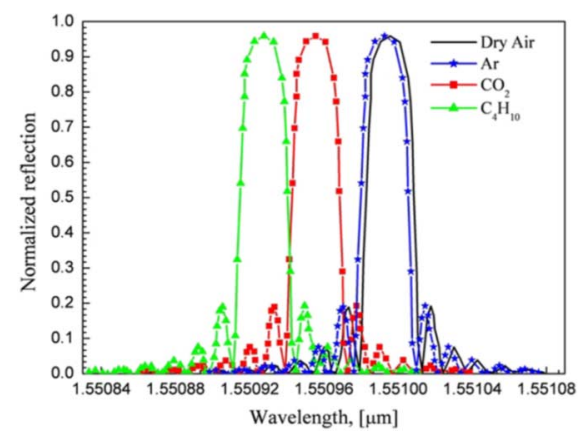

Fig. 3. Normalized reflection characteristics at $T=27^{\circ} \mathrm{C}$, of the proposed PCFG, for dry air (solid black line), argon (blue line, stars), carbon dioxide (red line, squares), and n-butane (green line, triangles), as a function of the wavelength. It is evident that for more dense gaseous solutions there is a measurable downward shift in the reflection spectrum, thus enabling the identification of the gas composition.

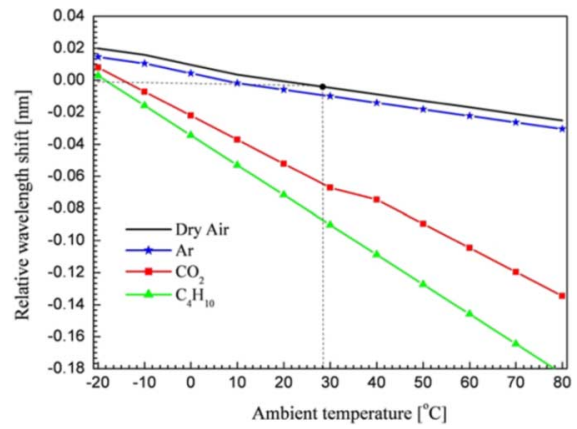

Fig. 4. Impact of the ambient temperature in Celsius to the relative wavelength shift in nanometers, of the reflection spectra of the proposed PCFG, for dry air (solid black line), argon (blue line, stars), carbon dioxide (red line, squares), and n-butane (green line, triangles). Observe the linear behavior in the relative shift.

in Fig. 3, we can conclude that for different gaseous solutions the spectral response of the PCFG is different, thus enabling us to use the measured spectra for the accurate identification of the gas flown in the place of the microstructured holes. Additionally, it was found that the maximum reflectivities are almost the same. The wavelength $\lambda_{\max }$, at which the maximum reflectivity occurs, is $1.550995 \mu \mathrm{m}$ for dry air, $1.550990 \mu \mathrm{m}$ for Ar, $1.550930 \mu \mathrm{m}$ for $\mathrm{CO}_{2}$, and $1.550903 \mu \mathrm{m}$ for $\mathrm{C}_{4} \mathrm{H}_{10}$. Each has a bandwidth of 4.241, 4.110, 3.938, and $3.872 \mathrm{GHz}$, respectively. Here, the bandwidth is defined as the width between the first zeros on either side of the maximum reflectivity. Further enhancement of the peak separations may be possible by incorporating thermoresponsive liquid solvents instead of gases, or incorporating gratings in solid-like polymer-infiltrated photonic bandgap fibers.

To estimate the impact of the ambient temperature variation in Celsius to the shift in nanometers of the $\lambda_{\max }$ relatively to dry air, in Fig. 4 we perform a thermooptical sensitivity analysis [12] of the relative wavelength shift (with respect to that of dry air at $T=27^{\circ} \mathrm{C}$ ), as a function of the ambient temperature, for the four different gases considered above. The shift increases as the mass density of the gas becomes larger. We can clearly see that the wavelength shift is almost linear without the lines, which correspond to different gases, intersect between each other. This simply means that in PCFGs the impact of the temperature fluc- tuation is uniform, a result that is found to be superior in comparison with the conventional FBGs [3], [13]. The reason is that the presence of the microstructured holes in the cladding significantly reduces the amount of silica material in comparison to conventional FBGs, thus reducing the thermal sensitivity of silica considerably. The main conclusion is that PCFGs can be potentially used as gas and/or liquid (fluidic) sensors. In addition, we saw that it is also possible to use the prescribed topology as a tunable optical filter. The technological feasibility of the grating inscribed into the PCF core can be achieved by using an ArF laser writing with a $193-\mathrm{nm}$ pulsewidth of about $15 \mathrm{~ns}$, operating at a repetition rate of $40 \mathrm{~Hz}$. The experimental validation of the device remains to be performed.

\section{CONCLUSION}

To summarize our work, we have numerically demonstrated the use of PCFGs as fluidic sensors (gas/liquid) or as tunable WDM filters. Perhaps one of the most interesting results reported here is that the ambient temperature variation influences uniformly the reflection spectra in contrast to the conventional FBGs [3], [13], thus enabling our proposed structure to operate over a wider temperature range without any cross interference of the reflection spectra corresponding to different gaseous solutions.

\section{REFERENCES}

[1] K. Hogari, S. Tetsutani, J. Zhou, F. Yamamoto, and K. Sato, "Optical-transmission characteristics of optical-fiber cables and installed optical-fiber cable networks for WDM systems," J. Lightw. Technol., vol. 21, no. 2, pp. 540-545, Feb. 2003.

[2] T. Erdogan, "Fiber grating spectra," J. Lightw. Technol., vol. 15, no. 8, pp. 1277-1294, Aug. 1997.

[3] S. Gupta, T. Mizunami, T. Yamao, and T. Shimomura, "Fiber Bragg grating cryogenic temperature sensor," Appl. Opt., vol. 35, pp. 5202-5205, Sep. 1996.

[4] J. Broeng, D. Mogilevtsev, S. E. Barkou, and A. Bjarklev, "Photonic crystal fibers: A new class of optical waveguides," Opt. Fiber Technol., vol. 5, pp. 305-330, Jul. 1999.

[5] W. Bock and T. Eftimov, "Polarimetric and intermodal interference sensitivity to hydrostatic pressure, temperature, and strain to highly birefringent optical fibers," Opt. Lett., vol. 18, pp. 1979-1981, Nov. 1993.

[6] G. Ghosh, Handbook of Thermo-Optic Coefficients of Optical Materials with Applications. San Diego, CA: Academic, 1997.

[7] T. Mito, S. Fujino, H. Takebe, K. Morinaga, S. Todoroki, and S. Sakaguchi, "Refractive index and material dispersions of multi-component oxide glasses," J. Non-Cryst. Solids, vol. 210, pp. 155-162, Aug. 1997.

[8] J. Owens, "Optical refractive index of air: Dependence of temperature, pressure and composition," Appl. Opt., vol. 6, pp. 51-59, Jan. 1967.

[9] D. P. Shelton and V. Mizrahi, "Refractive index dispersion of gases measured by optical harmonic phase matching," Phys. Rev. A, vol. 33, pp. 72-76, Jan. 1986.

[10] C. Kerbage, P. Steinvurzel, P. Reyes, P. S. Westbrook, R. S. Windeler, A. Hale, and B. J. Eggleton, "Highly tunable birefringent microstructured optical fibers," Opt. Lett., vol. 27, pp. 842-844, May 2002.

[11] N. Florous and M. Koshiba, "Virtual boundary method for the analysis of elliptically cross-sectional photonic crystal fibers with elliptical pores," J. Lightw. Technol., vol. 23, no. 8, pp. 1763-1773, Apr. 2005.

[12] N. J. Florous, S. K. Varsheney, K. Saitoh, and M. Koshiba, “Thermooptical sensitivity analysis of highly birefringent polarimetric sensing photonic crystal fibers with elliptically elongated veins," IEEE Photon. Technol. Lett., vol. 18, no. 8, pp. 1663-1665, Aug. 2006.

[13] O. Frazão, J. P. Carvalho, L. A. Ferreira, F. M. Araújo, and J. L. Santos, "Discrimination of strain and temperature using Bragg gratings in microstructured and standard optical fibres," Meas. Sci. Technol., vol. 16, pp. 2109-2113, Oct. 2005. 